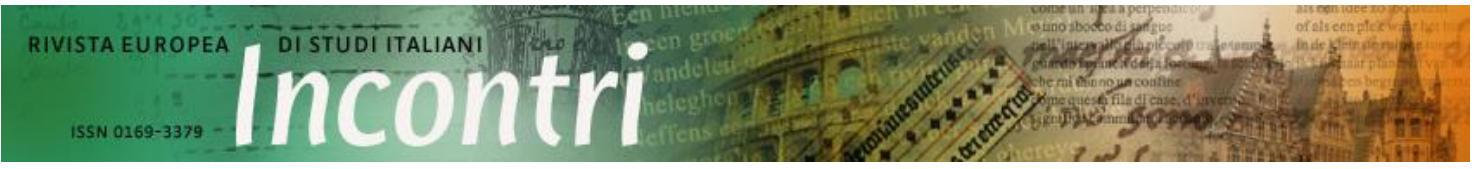

Anno 35, 2020 / Fascicolo 1 / p. 46-58 - unw.rivista-incontri.nl - http://doi.org/10.18352/incontri.10333 (C) The author(s) - Content is licensed under a Creative Commons Attribution 3.0 Unported License Publisher: Werkgroep Italië Studies, supported by Utrecht University Library Open Access Journals

\title{
Leonardo industrial designer
}

\section{Sara Taglialagamba*}

Per Leonardo, l'arte è un'imitazione del mondo reale poiché si fonda sulla comprensione scientifica delle leggi che governano la natura. Egli cerca di individuare la presenza di "forme matrici" che governano la varietà di tutte le cose. ${ }^{1}$ Queste sono realtà archetipe modulabili all'infinito secondo regole geometriche e di proporzione. In tal modo, egli sembra volere ritrovare tratti di perfezione matematica al mondo delle forme naturali e caratteri plastici al mondo astratto delle forme geometriche. È dunque possibile parlare di una 'grammatica delle forme'2 che funziona per una serie di rapporti proporzionali, ripetizioni, simmetrie e analogie concettuali, capaci di generare immagini concrete di estrema bellezza.

Ad appassionare Leonardo, oltre alle forme rotonde, ${ }^{3}$ alle forme combacianti $i^{4} \mathrm{e}$ alle forme ramificate, ${ }^{5}$ sono gli intrecci ${ }^{6}$ - forme matrici dinamiche ad alto potenziale

\footnotetext{
* Questo mio saggio nasce come un successivo approfondimento del mio intervento al seminario organizzato da Pierre Caye e Francesco Solinas Léonard et l'ornement presso il Collège de France il 14 novembre 2015 poi edito come 'Machines et ornement chez Léonard', in: P. Caye \& F. Solinas (a cura di), Les Cahiers de l'ornement. 1, Roma, De Luca, 2016, pp. 45-67. Il tema vedrà l'edizione di un libro in corso di stampa e una mostra organizzata dal Distretto Turistico Venezia Orientale a settembre 2020. Per un'introduzione alla storia del Design rimando a: R. De Fusco, Storia del design, Bari, Laterza, 1985 ; A. Pansera, Storia del design industriale italiano, Bari, Laterza, 1993; G. D’Amato, Storia del design, Milano, Mondadori, 2005; B. Buerdek, Design. Storia, teoria e pratica del design del prodotto, Roma, Mondadori, 2008; M. Vitta, Il progetto della bellezza. Il design tra arte e tecnica del 1851 ad oggi, Torino, Einaudi, 2011.

1 Per una definizione più approfondita del termine di “matrici universali" e per la loro classificazione rimando a D. Laurenza, 'Le forme come matrici universali', in: P. Galluzzi (a cura di), La mente di Leonardo: nel laboratorio del genio universale, catalogo della mostra (Firenze, Galleria degli Uffizi, 28 marzo 2006 - 7 gennaio 2007), Firenze, Giunti, 2006, pp. 188-201.

2 D. Laurenza, 'La grammatica delle forme: proporzione e analogia', in: Galluzzi, La mente di Leonardo, cit., pp. 151-157; P. Galluzzi \& D. Laurenza, 'L'equilibrio delle forme', in: ivi , pp. 166-177; F. Camerota \& D. Laurenza, 'Trasformazioni e metamorfosi', in: ivi, pp. 178-187. Rimando anche al link a cura del Museo Galileo di Firenze e relativo a un approfondimento a cui ho rimandato nelle mie note bibliografiche relative al catalogo della mostra sopracitata La mente di Leonardo (2006): https: / /brunelleschi.imss.fi.it/menteleonardo/imdl.asp?c=13419\&k=13363\&rif=13367 (30 giugno 2020).

3 Per esempio: Studi per una chiesa a pianta centrale, c. 1487-1490, Manoscritto B, f. $95 \mathrm{v}$, cfr. https://www.leonardodigitale.com/sfoglia/manoscritto-b-dell-institut-de-france/0095-v/ (30 giugno 2020).

4 Per esempio: Studio anatomico di un feto, c. 1511, Windsor, RL 19102 r, cfr. https: / / www.rct.uk/collection/919102/recto-the-fetus-in-the-womb-verso-notes-on-reproduction-withsketches-of-a-fetus (30 giugno 2020).

5 Per esempio: Studi anatomici della muscolatura della spalla, c. 1510-1511, Windsor, RL 19015 r, cfr. https: / /www.rct.uk/collection/search\#/30/collection/919015/recto-the-muscles-of-the-upper-spineverso-the-muscles-of-the-upper-spine (30 giugno 2020).

6 Per esempio: Schema ad albero tratto dal Summa de Arithmetrica di Luca Pacioli per illustrare $i$ vari tipi di proporzioni, c. 1503-1505, Codice di Madrid II, f. 78 r, cfr. https://www.leonardodigitale.com/ sfoglia/madrid-II/0078-r/ (30 giugno 2020).
} 
decorativo. Leonardo ricorre alle forme intrecciate per descrivere fenomeni diversi tra loro. In natura le utilizza per descrivere i capelli ${ }^{7}$ e gli studi d'acqua, ${ }^{8}$ nella tecnica per le centine architettoniche ${ }^{9}$ e nelle elaborazioni ludiche per gli intrecci dei simbolici nodi vinciani ${ }^{10}$ e per le trame di foglie, radici, rami, nastri svolazzanti e cordicelle dorate nella vegetazione nella Sala delle Asse. ${ }^{11}$ Pur se diversi tra loro, tutti questi elementi sono sottoposti alla lente della forma matrice a intreccio, come se questa fosse un modo di ingabbiare la realtà, studiarla e restituirla priva dei suoi segreti. Con la stessa facilità e efficacia, le forme ondulate descrivono l'artificiosa acconciatura della Leda e il gorgheggiare dell'acqua, al pari delle trame decorative dei nodi vinciani e dei nastri tra gli alberi della Sala delle Asse.

Le forme matrici hanno pertanto una doppia funzione: sono sia un metodo di investigazione per meglio comprendere il mondo naturale, sia uno strumento di progettazione artistica e tecnica. L'ornato che ne consegue dunque non è un orpello visivo, la decorazione accessoria di un'opera, quanto piuttosto una parte indivisibile dal tutto e per questo necessaria e funzionale. La frase celeberrima appuntata in un foglio del Codice Atlantico, 'Non po' essere bellezza e utilità, come appare nelle fortezze e nelli uomini?' , ${ }^{12}$ pur se rivolta ad altro, è utile a spiegare tale equazione bellezza-utilità ispirata dalla definizione platonica dell'Ippia Maggiore per cui 'bello' è da intendersi ciò che è conveniente e utile. Da parte loro, le pitture di Leonardo non facevano eccezione e offrivano esempi di straordinaria bellezza e perfezione: in esse l'ornamento non è un elemento sovrapponibile e accessorio, esso è parte integrante dei concetti espressi in pittura. ${ }^{13}$ L'ambiente di formazione proponeva esempi importanti che si risolvevano in forme decorative di grande impatto. Andrea Verrocchio,

\footnotetext{
${ }^{7}$ Cfr. Testa di Leda, c. 1505-1508, Windsor, RL 12518, cfr. https://www.rct.uk/collection/912518/thehead-of-leda (30 giugno 2020).

8 Per esempio: Studi sul movimento dell'acqua, c. 1510-1512, Windsor, RL $12660 \mathrm{v}$, cfr. https: / /www.rct.uk/collection/912660/studies-of-water (30 giugno 2020).

9 Studi di volte architettoniche, c. 1515, Codice Atlantico, f. 200 r-a $\left[\begin{array}{lllll}537 & \text { r }\end{array}\right]$ cfr. https://www.leonardodigitale.com/sfoglia/codice-atlantico/0537-r/ (30 giugno 2020).

10 Cfr. https://www.britishmuseum.org/collection/object/P_1877-0113-364 (30 giugno 2020).

11 Rimando alle immagini ufficiali della volta con intrecci arborei e nastri: https://www.milanocastello.it /it/content/leonardo-da-vinci (30 giugno 2020).

12 La frase 'Non po' essere bellezza e utilità, come appare nelle fortezze e nelli uomini' è appuntata sul foglio 147 r-b [399 r] del Codice Atlantico (c. 1490-1491). Si veda: C. Pedretti, Studi vinciani: documenti, analisi e inediti leonardeschi, Genève, Droz, 1957, p. 31, tav. 9. Grazie a una felice intuizione di P.C. Marani ( $L$ 'architettura fortificata negli studi di Leonardo da Vinci: con il catalogo completo dei disegni, Firenze, Giunti, 1984, p. 291) questa celeberrima frase di Leonardo, considerata da molti studiosi una negazione - si veda The Literary Works of Leonardo da Vinci, compiled and edited from the original manuscripts by J.P. Richter, London, Low, 1883, § 1445; A.M. Brizio, Scritti scelti di Leonardo da Vinci, Torino, Utet, 1952, p. 238 e lo stesso C. Pedretti (Leonardo architetto, Milano, Electa, 1978, p. 156) sarebbe invece da considerare in tono interrogativo. Prima di lui, anche I. Calvi (L'architettura militare di Leonardo da Vinci, Milano, Libreria Lombarda, 1943, p. 18) aveva proposto che la frase potesse avere un'accezione interrogativa anche se reputava che dovesse leggersi in questo modo: 'Non po' essere bellezza e utilità? come appare nelle fortezze e nelli uomini' e, dunque, l'interrogazione avrebbe riguardato solo la prima parte della frase. Marani, anche a seguito dell'approvazione del filologo italiano Ettore Casamassima, propose di leggere l'intera frase in forma interrogativa: 'Non po' essere bellezza e utilità, come appare nelle fortezze e nelli uomini?'. Fu Pedretti ad accettare con entusiasmo questa proposta, che, va ben sottolineato, presupporrebbe così una risposta di tipo assertiva, proponendo anche di leggere la frase come una postilla da riferire alla lettura da parte di Leonardo di un passaggio del De Civitate Dei di Sant'Agostino (XXII, 24) - libro posseduto da Leonardo - nella sua lectio doctoralis tenuta all'Università di Ferrara nel 1992 con il titolo di Il concetto di bellezza e utilità in sant'Agostino $e$ Leonardo, parzialmente pubblicata in C. Pedretti, 'Il concetto di bellezza e utilità in Sant'Agostino e Leonardo', in: Achademia Leonardi Vinci, 5 (1992), pp. 107-111.

${ }^{13}$ Sul rapporto tra ornamento e pittura in Leonardo rimando al contributo di P.C. Marani al convegno a Parigi, poi pubblicato con il titolo di: “"Admirable... par sa gracieuse simplicité, par son élégance modeste sans aucune odieuse surcharge": Léonard et la question de l'ornement', in: Caye \& Solinas, Les Cahiers de l'ornement, cit., pp. 74-92.
} 
'scultore, orafo, intagliatore, pittore e musico perfettisimo', il recupero dell'antico e il serpeggiare di una certa aria umbra, di cui era permeato anche l'ambiente fiorentino avrebbero condotto a un disegno come il volto del Guerriero del British Museum di Londra. ${ }^{14}$ L'ornamento dunque tende a rispondere ai criteri di ordine, bellezza e invenzione.

Ė interessante rilevare che Leonardo avrebbe potuto tenere in mente opere che già, nello stesso modo, potevano incarnare lo stesso binomio bellezza-utilità. Tra il XV e il XVI secolo, Firenze era diventata una città rinomata non solo per i suoi artisti ma anche per i suoi numerosi artigiani. Questi ultimi erano maestri abili a produrre una grande varietà di prodotti grazie non soltanto alle loro straordinarie abilità, ma anche alla loro preparazione culturale e al lavoro realizzato spesso in sinergia con gli stessi artisti. Infatti, la polivalenza delle botteghe gestite dagli artisti di maggiore levatura determinava la convivenza tra arti come la pittura, la scultura e l'architettura con quelle più manuali come l'intaglio, l'oreficeria, i disegni per vetrate e ricami, i mosaici e le illustrazioni di libri. Questo divario tra artisti e artifices nel 1546 sarà teorizzato nel passo 'Sulla maggioranza e nobiltà delle arti' da Benedetto Varchi ma senza che tale teorizzazione sancisse ancora una rigida emarginazione sociale, solo una suddivisione per così dire "ideale" della differenza che da sempre era intercorsa tra le arti liberali e quelle meccaniche. ${ }^{15} \mathrm{~A}$ dare impulso a questo fenomeno di produzione di oggetti di uso a Firenze fu la straordinaria crescita dell'economia fiorentina che non ebbe rivali nelle altre città europee grazie alla vigorosa attività della banca, del commercio e della produzione tessile. Tale successo determinò la crescita di un consistente ceto medio-alto sempre più orientato a un consumo di lusso dal gusto raffinato e elegante sulla scia di Lorenzo il Magnifico e che seppe rimanere tale anche dopo la sua scomparsa. Tale domanda determinava necessariamente la richiesta di una manodopera specializzata non solo agli artisti (si ricordino le magnifiche creazioni degli orafi del tempo tra cui, oltre agli anonimi, è possibile annoverare Lorenzo Ghiberti, Brunelleschi, Donatello, Maestro Gualandi, Francesco d'Antonio, Giusto da Firenze ma anche $\mathrm{i}$ ricami, $\mathrm{i}$ tessuti, i lampassi e i paramenti realizzati dalle manifatture fiorentine e lucchesi su disegno di Paolo Schiavo, Antonio del Pollaiolo e Sandro Botticelli) ma anche agli artigiani al tempo attivi nelle vivaci botteghe fiorentine dei Pollaiolo e del Verrocchio. ${ }^{16}$ Ad alimentare questa crescita economica si registra una maggiore disposizione di ricchezza e anche un'apertura a nuovi tipi di oggetti da parte del mercato che, insieme ai mutamenti della moda, coinvolsero le élites del tempo non soltanto nella committenza di grandi opere d'arte ma anche in tutte le arti cosiddette minori. Accanto all'oreficeria, per certi versi ritenuta la maggiore delle arti minori, è possibile includere libri miniati e decorati, gli abiti, gli oggetti tradizionali molto spesso appartenenti alla sfera religiosa, la decorazione d'interni, l'arredamento delle abitazioni e perfino il collezionismo di strumenti scientifici che soddisfacessero contemporaneamente gli interessi per la tecnologia e per la produzione artigianale. Tutto questo mondo di opere d'arte e manufatti

\footnotetext{
${ }^{14}$ Cfr. https://www.britishmuseum.org/collection/object/P_1895-0915-474 (30 giugno 2020).

15 Indico come contributi fondamentali: R.A. Goldthwaite, 'Realtà economico-sociale e status culturale dell'artigianato', in: F. Franceschi \& G. Fossi (a cura di), La grande storia dell'artigianato: arti fiorentine. II: il Quattrocento, Firenze, Cassa di Risparmio di Firenze-Giunti, 1999, pp. 9-26; F. Franceschi, 'La bottega come spazio di sociabilità', in: ivi, pp. 65-84; R. Mancini, 'Il principe e l'artigiano: propositi d'emarginazione sociale nella Firenze del Cinquecento', in: F. Franceschi \& G. Fossi (a cura di), La grande storia dell'artigianato: arti fiorentine. III: il Cinquecento, Firenze, Cassa di Risparmio di Firenze-Giunti, 2000, pp. 25-40; 'La civiltà degli utensili' in: C. Cartier et al. (a cura di), Antichi strumenti e utensili dalla collezione Nessi, Milano, 5 Continents, 2004, pp. 133-206.

16 È necessario citare almeno un libro fondamentale: G. Cantelli (a cura di), Storia dell'oreficeria $e$ dell'arte tessile in Toscana dal medioevo all'età moderna, Firenze, Banca Toscana, 1996, in particolare pp. 79-159.
} 
artigianali divenne ben presto oggetto degli appassionati racconti di ambasciatori e viaggiatori incantati dalle produzioni versatili degli artisti e degli artigiani locali. Anche Leonardo poteva guardare a queste tipologie in particolar modo quelle schizzate nei taccuini di bottega o nei libri di artista che soddisfacevano senza dubbio la richiesta di un oggetto bello e utile al tempo stesso, ma un unicum e quindi non esattamente riproducibile con una certa facilità. Ora con questi assunti, proviamo ad analizzare tre casi che offrono riprove calzanti e estremamente pertinenti.

\section{Il "ludo geometrico": astrazioni geometriche di grande raffinatezza}

Leonardo sottopone sia le forme organiche sia le forme geometriche a un processo di astrazione riconducendo le loro strutture a una matrice geometrico-matematica dal valore di ornato. Per questo lo schema di un dispositivo tecnologico, ${ }^{17}$ che più che una ruota d'acqua sembra una costruzione geometrica, è stato definito da Pietro Marani un 'bellissimo disegno che s'avvolge in un moto centrifugo'. ${ }^{18}$ Allo stesso modo, Leonardo tende a condurre a forme decorative triangolari e semilunari i lembi della valvola cardiaca, ${ }^{19}$ strutture simili si osservano in disegni squisitamente geometrici come i disegni di lunulae. ${ }^{20}$ II "ludo geometrico" si traduce qui in un motivo decorativo di grande raffinatezza, paragonabile soltanto alla ricercata eleganza e all'estremo virtuosismo prospettico dei diagrammi di solidi regolari eseguiti per l'amico Luca Pacioli come illustrazioni del De Divina Proportione, pubblicato a Venezia nel 1509.

Dalle strutture geometriche, ai solidi, ai fogli tecnologici il passo è breve. Il punto più alto delle astrazioni geometriche a forma decorativa è l'esatta rappresentazione del solido geometrico detto mazzocchio. Il termine mazzocchio indicava comunemente il copricapo rotondo molto diffuso al tempo ma, dal punto di vista geometrico, il mazzocchio può essere definito come un anello poligonale a sezione solitamente esagonale o ottagonale. Fu Piero della Francesca a parlare nella proposizione IV del terzo libro del De prospettiva pingendi del problema geometrico che sottintende il mazzocchio o 'torculo' come lui lo chiama: 'Il torculo dato avente octo circuli continente la grossezza, in dodici parti uguali deviso, con proporzione deminuire'.

Proprio per la sua forma, fu uno degli oggetti più difficili da rappresentare in prospettiva e pertanto utilizzato come indice di talento nell'esercizio di disegno per gli scolari che - come di consuetudine - svolgevano l'apprendistato nelle botteghe degli artisti. Si ritrova dipinto nelle opere di Paolo Uccello, nelle più belle tarsie lignee del Rinascimento, nelle lezioni di prospettiva di Lorenzo Serigatti e perfino nell'arte contemporanea, come accade in due grandi e celebri mazzocchi, quello marmoreo appartenente all'istallazione unica di Mimmo Paladino all'Ara Pacis ${ }^{21}$ e quello metallico addossato alle mura di Porta Frascati nella città di Prato realizzato da Ben Jakober e Yannick Vu nel 1994. ${ }^{22}$ Così come quello di Piero ovvero un toro a sezione ottagonale, quello disegnato da Leonardo è formato da due anelli spiraliformi

\footnotetext{
17 Progetto per uno strumento meccanico, c. 1487-1490, Codice Atlantico, f. $221 \mathrm{v}$-a [598 i-r], cfr. https://www.leonardodigitale.com/sfoglia/codice-atlantico/0598-r/ (30 giugno 2020).

${ }^{18}$ Marani, L'architettura fortificata, cit., p. 134.

${ }^{19}$ Rimando al particolare dei lembi a forma semilunare delle valvole del cuore in Studi per le valvole cardiache, schizzo per una sedia, diagrammi e calcoli, c. 1515, Windsor, RL $19079 \mathrm{v}$, cfr. https: / /www.rct.uk/collection/919079/studies-of-the-valves-of-the-heart-and-sketch-of-a-suditary-andcalculations (30 giugno 2020).

20 Studi di lunulae, c. 1515, Codice Atlantico, f. 167 r-b [455 r], cfr. https://www.leonardodigitale.com /sfoglia/codice-atlantico/0455-r/ (30 giugno 2020).

${ }^{21}$ Achille Bonito Oliva, Federica Pirani \& James Putnam (a cura di), Mimmo Paladino Brian Eno. Opera per l'Ara Pacis, catalogo della mostra (Roma, Ara Pacis, 11 marzo-1 giugno 2008), Prato, Gli Ori, 2008.

22 Cfr. http://www.pratoturismo.it/it/cosa/luoghi-da-vedere/Arte-contemporanea-e-street-art/ mazzocchio/ (30 giugno 2020).
} 
intrecciati e si inspira alla ruota dentata ad andamento a spirale per un meccanismo di orologeria. ${ }^{23}$ Ciascun anello della struttura serpentinata è formato da una serie di segmenti, risultanti dalla torsione di un comune mazzocchio a sezione ottagonale. Sotto si legge la frase: 'Corpo nato della prospettiva di Leonardo Vinci, discepolo della sperienza' e la spiegazione 'Sia fatto questo corpo sanza esemplo d'alcun corpo, ma solamente con semplici line', che, nell'atto di svolgersi come un serpente, riassume i cardini delle fondazioni scientifiche dello studio dell'arte - prospettiva, proporzione e meccanica - alle quali Leonardo si rivolgeva fin dai primi tempi del momento del suo soggiorno milanese. L'ornato in questo caso è strettamente funzionale perché connaturato alla struttura stessa.

Un altro caso esemplare è costituito dai progetti di “macchine" per Ludovico il Moro. In età sforzesca, l'industria tessile era molto sviluppata e il commercio di tessuti di lusso aveva una grande importanza per l'economia del Ducato per il quale Leonardo progetta numerose macchine utensili automatizzate che sfruttano l'energia dell'acqua. É utile osservare la successione di tre disegni, databili tra il 1495 e il 1496, il $31 \mathrm{v}-\mathrm{b}$ $\left[86\right.$ r] ${ }^{24}$ (c. 1495), il $57 \mathrm{r}$-a [159 i r] ${ }^{25}$ (c. 1495) e il $318 \mathrm{v}$-a $[874 \mathrm{v}]^{26}$ (datato 2 gennaio 1496), nei quali Leonardo raffigura parte di una macchina per la produzione di spilli ${ }^{27}$ concentrandosi sulla soatta ovvero la cinghia di trasmissione. L'intenzione di base più che studiare il particolare del meccanismo sembrerebbe quella di porre l'attenzione sul dettaglio ornamentale della cinghia, per poi concentrarsi sull'aspetto tecnico. L'aspetto decorativo doveva interessarlo moltissimo in questo periodo. Apparteneva al verso del primo foglio, ovvero il $31 \mathrm{~V}-\mathrm{b}$ [86 r] (c. 1495), il frammento RL 12282A $\mathrm{A}^{28}$ (anch'esso c. 1495), oggi conservato a Windsor e considerato un cosiddetto foglio di rattoppo di Pompeo Leoni che, come noto, restaurò anche le parti da cui erano stati asportati i disegni. Il piccolo disegno araldico appartenente alla casata Sforza raffigura due dragoni sibilanti arrotolati attorno a due bastoni incrociati, con le lettere GM[S] da riferire a Galeazzo Maria Sforza. Ritornano le forme matrici ondulate, creando un'immagine simile al corpo geometrico del mazzocchio a sezione ortogonale.

Questa sezione offre l'occasione di parlare di Leonardo industrial designer ${ }^{29}$ ovvero come progettista di oggetti d'uso, di piccole dimensioni, in funzione della loro riproducibilità e della ricerca dei materiali per la loro produzione in serie come utensili, cappelli, oggetti di uso quotidiano, occhiali. È in questo campo che l'ornato prende il sopravvento pur rispondendo alle caratteristiche di base: bellezza, utilità, invenzione. Ne Le memorie de fatti di Leonardo da Vinci a Milano, scritte dopo il 1631, Giovanni Ambrogio Mazenta afferma che 'nelle botteghe d'Arte, molte machine s'usan ritrovate

\footnotetext{
23 Forma nata dalla prospettiva ed altri studi, c. 1490, Codice Atlantico, f. 191 r-a [520 r], cfr. https://www.leonardodigitale.com/sfoglia/codice-atlantico/0520-r/ (30 giugno 2020).

${ }^{24}$ Cfr. https: //www.leonardodigitale.com/sfoglia/codice-atlantico/0086-r/ (30 giugno 2020).

${ }^{25}$ Cfr. https: / /www.leonardodigitale.com/sfoglia/codice-atlantico/0159-r/ (30 giugno 2020).

${ }^{26} \mathrm{Cfr}$. https: //www.leonardodigitale.com/sfoglia/codice-atlantico/0874-v/ (30 giugno 2020).

27 È da precisare che la macchina è disegnata anche ai fogli 25 r-b [74i-r] e 25 r-a [74ii-r] (c. 1495) dove Leonardo pone attenzione a tutto l'assemblaggio strutturale. Rimando a C. Pedretti, The Codex Atlanticus of Leonardo da Vinci: A Catalogue of Its Newly Restored Sheets, New York, Phaidon, 1978-1979, I, pp. 56 e 61 con i rimandi relativi.

28 Cfr. https://www.rct.uk/collection/970124/recto-an-emblem-of-galeazzo-maria-sforza-verso-afragment-of-a-design-for-a-needle (30 giugno 2020).

29 La stupenda definizione di Leonardo industrial designer fu introdotta per la prima volta da Carlo Pedretti e a lui, ancora una volta, devo l'incoraggiamento di perseguire anche questo filone di studio e ricerca con il rigore filologico, l'attenzione meticolosa, la precisione chirurgica e l'entusiasmo contagioso che lui stesso ha magistralmente saputo trasmettermi e insegnarmi. Si veda: Pedretti, Leonardo architetto, cit., pp. 324-344. Si veda anche il mio articolo: 'Leonardo designer alla corte di Milano. Ordine, bellezza e invenzione', in: Art Dossier, 1 (2015), pp. 60-65.
} 
da Leonardo per tagliar, lustrar christalli, ferri, pietre ${ }^{30}$ e che il celebre Annibale Fontana 'scultore di cammei, christalli, gioie, marmi [...] professava d'haver da le cose di Leonardo appreso quanto sapeva'. ${ }^{31}$ Nel Tempio della pittura edito nel 1590, Giovanni Paolo Lomazzo conferma che l'intagliatore Giovanni Antonio Maggiore 'ritrovò l'arte d'intornir gli ovati, che è cosa degna di molta meraviglia, la quale fu poi insegnata da un discepolo del Melzi a Dionigi, fratello del Maggiore, che la esercita ora felicissimamente'. ${ }^{32}$ Come osserva Paola Venturelli, l'influsso documentato di Leonardo sulle arti preziose in ambiente lombardo è quindi considerevole. ${ }^{33}$ Essenziale fu la vivace situazione del Ducato durante entrambi i soggiorni milanesi quando si registra il massimo sviluppo dei prodotti suntuari - tra cui broccati, prodotti auroserici, armi, smalti, oggetti preziosi - che costituivano le merci trainanti dell'economia locale. La pittura era destinata a rimanere nella stessa posizione in cui Leonardo l'aveva relegata nella famosa lettera di presentazione a Ludovico il Moro: ovvero all'ultimo posto. Leonardo era catturato da un continuo fervore inventivo. Alcuni disegni con studi di motivi di ornato sono, infatti, datati post 1485: tra questi, lo studio per il piatto di una legatura di un libro, ${ }^{34}$ le decorazioni per mattonelle, ${ }^{35}$ medaglie e insegne $^{36}$ forse da utilizzare come emblemi, fino ai disegni più tardi con lo studio per un soffitto o un pavimento ${ }^{37}$ a tarsia o a intreccio. ${ }^{38}$

Ad avere la massima fortuna e inventiva furono senza dubbio i gioielli. ${ }^{39}$ Come attestano le fonti, per migliorare la loro lavorazione sembra che Leonardo progettasse dispositivi specializzati per lucidare le pietre, tagliarle, sagomarle: tra queste macchine, ad esempio, si trovano una macchina per 'forare cristalli' al foglio $23 \mathrm{v}$ del Manoscritto $\mathrm{I}^{40}$ (c. 1497-1499), un tornio e alcuni 'mulinelli da gioie' per la loro lavorazione muniti di ruote dentate e rocchetti entrambi al foglio $384 \mathrm{v}-\mathrm{b}[1063 \mathrm{v}]^{41}$ del Codice Atlantico interamente ricoperto anche da studi di macchine militari (c. 1487). Anche i materiali e la loro conoscenza furono essenziali: Leonardo si

${ }^{30}$ Giovanni Ambrogio Mazenta. Alcune memorie dei fatti di Leonardo da Vinci a Milano e dei suoi libri, ripubblicate e illustrate da D.L. Gramatica, Milano, La Vita Felice, 2008, p. 63.

31 Ibidem.

32 R.P. Ciardi (a cura di), Gian Paolo Lomazzo. Scritti sulle arti, Firenze, Marchi \& Bertolli, 1973-1974, vol. 1, p. 259.

33 Sul rapporto tra Leonardo, i gioielli e le arti preziose rimando ai contributi di P. Venturelli in: Leonardo da Vinci e le arti preziose: Milano tra XV e XVI secolo, Venezia, Marsilio, 2002 e agli ultimi aggiornamenti La moda alla corte degli Sforza. Leonardo da Vinci tra creatività e tecnica, Cinisello Balsamo, Silvana Editoriale, 2019; Arte orafa milanese. Maestri, botteghe, tecniche e prodotti (1450-1527). Con Leonardo da Vinci tra creatività e tecnica, Cinisello Balsamo, Silvana editoriale, 2020.

34 Studio per il piatto per la legatura di un libro, c. 1483-1485, Torino, Biblioteca Reale, inv. 15572 v

35 Progetto per mattonella, c. 1497-1499, Codice Atlantico, f. 261 r-b [701 r], cfr. https://www.leonardodigitale.com/sfoglia/codice-atlantico/0701-r/ (30 giugno 2020).

36 Medaglioni e emblemi, c. 1508, Codice Arundel, f. 225 r, cfr. https://www.leonardodigitale.com /sfoglia/codice-arundel/0225-r/ (30 giugno 2020).

37 Intrecci, c. 1497-1499, Codice Atlantico, f. 261 r-a [700 r], cfr. https://www.leonardodigitale.com /sfoglia/codice-atlantico/0700-r/ (30 giugno 2020).

${ }^{38} \mathrm{Mi}$ riferisco in ordine ad alcuni particolari decorativi che si trovano rispettivamente in: per lo studio del piatto della legatura di un libro: Torino, Biblioteca Reale, inv. 15572 v (c. 1483-1485); per lo studio di mattonella: CA, f. 261 r-b [701 r] (c. 1497-1499); per gli studi di medaglie: Codice Arundel, f. 225 r, (c. 1508); per lo studio di un soffitto o di pavimentazione: CA, f. 261 r-a [700 r] (c. 1497-1499).

${ }^{39}$ Segnalo in via di pubblicazione questi contributi di P. Venturelli: 'Medaglie ed enseignes a Milano. Leonardo da Vinci e dintorni (tra Caradosso e Roma)', in: Oltre l'ornamento. Il gioiello tra identità, lusso e moderazione, Giornata di Studio Internazionale, Padova 22 febbraio 2019, Atti in corso di pubblicazione (in OADI, Osservatorio delle Arti Decorative in Italia); 'Lustro' $e$ 'trasparente'. Leonardo da pietre dure e materiali per le arti preziose, in: M. Quaglino, C. Vecce et alii (a cura di), Leonardo e gli scritti di pittura in Europa: fonti e ricezioni, Convegno Internazionale (Parigi-Torino), II Sessione Torino 27-29 novembre 2019, in corso di pubblicazione.

$40 \mathrm{Cfr}$. https://www.leonardodigitale.com/sfoglia/manoscritto-i-dell-institut-de-france/0023-v/ (30 giugno 2020).

${ }_{41}$ Cfr. https: / / www.leonardodigitale.com/sfoglia/codice-atlantico/1063-v/ (30 giugno 2020). 
interessa non solo alle pietre ma anche all'avorio. La sua conoscenza doveva essere molto approfondita se proprio nel 1500 è chiesta a lui una consulenza circa alcuni vasi 'di pietre dure, zoè: diaspise, christallo et anitista' richiesta da Isabella Gonzaga in una lettera del 3 maggio 1502 e ricevendo comunicazione dall'agente fiorentino Francesco Malatesta in data 12 maggio che Leonardo aveva accordato la sua preferenza per la screziatura e la trasparenza del vaso di ametista perché 'chosa nuova et per diversità de cholori mirabile'. Leonardo si interessò anche ad alcuni metodi sperimentali per ottenere nuovi materiali come le pietre dure, il vetro, il calcedonio e perfino alcuni segreti per produrre 'perle grosse' ottenendo una pasta da perle piccole grazie a una mistione con albume d'uovo e acido citrico. ${ }^{42}$

I viluppi decorativi, spesso aniconici ma dotati di una inventiva esuberante, costituiscono l'ossatura per le creazioni orafe. Il piglio da orafo si ritrova nelle decorazioni progettate per lavori su metallo: la sua fantasia crea molti schizzi di intrecci metallici per braccialetti ${ }^{43}$ e medaglioni, come il magnifico pendente in un foglio perduto (già Oxford, Christ Church). L'estrema raffinatezza di questi oggetti sembra addirittura anticipare gli artisti delle Arts and Crafts dove ornato e supporto coincidono. Rispondono a questa vocazione anche $\mathrm{i}$ compassi finemente decorati disegnati durante il secondo soggiorno milanese, come quelli al foglio $177 \mathrm{v}-\mathrm{c}$ [484 r] ${ }^{44}$ (c. 1493-1494) oppure quelli al foglio 14 r-b [48 r] ${ }^{45}$ (c. 1513), entrambi appartenenti al Codice Atlantico, e in particolar modo nel periodo tardo, quando forse Leonardo si trovava già in Francia, così come schizza al foglio $259 \mathrm{r}$-a $[696 \mathrm{r}]^{46}$ (c. 1515), anch'esso appartenente al Codice Atlantico, molto diversi da quelli precoci ad apertura assestabile che Leonardo studia a Milano alla pagina $108 \mathrm{v}$ del Manoscritto $\mathrm{H}^{47}$ (c. 1494), al tempo semplici strumenti di misurazione.

Come "apparatore di feste" si occupò anche di costumi, tessuti originali e accessori diversi. ${ }^{48}$ A Milano, sia per la fastosa corte degli Sforza sia per i committenti francesi che inaugurarono il suo secondo soggiorno milanese, per esempio, delinea intrecci decorativi e bordature, come nel caso del famoso foglio $68 \mathrm{v}$-b [190 v] ${ }^{49}$ (c. 1508) con il celebre disegno dello studio del motivo del calandrino - il piccolo uccello in gabbia con il motto 'I pensieri si voltano alla speranza'. Accanto alla gabbia, Leonardo schizza numerose bordature a filo, adatte probabilmente a essere tradotte in metallo prezioso per essere poi applicate agli abiti secondo la moda cortigiana diffusa al tempo. Rientra in questo gruppo anche il disegno di un dispositivo a martello

\footnotetext{
42 Venturelli, Leonardo da Vinci e le arti preziose, cit. pp. 105-111 con bibliografia relativa.

43 In basso nell'angolo a sinistra: Studi di intrecci metallici per un braccialetto a fascia, c. 1508, Codice Atlantico, f. 83 v-b [226 v], cfr. https://www.leonardodigitale.com/sfoglia/codice-atlantico/0226-v/ (30 giugno 2020).

44 https: / /www.leonardodigitale.com/sfoglia/codice-atlantico/0484-r/ (30 giugno 2020).

45 https: / /www.leonardodigitale.com/sfoglia/codice-atlantico/0048-r/ (30 giugno 2020).

46 https: //www.leonardodigitale.com/sfoglia/codice-atlantico/0696-r/ (30 giugno 2020).

$47 \mathrm{Cfr}$. https://www.leonardodigitale.com/sfoglia/manoscritto-h-dell-institut-de-france/0108-v/ (30 giugno 2020).

48 Rimando al mio contributo perché utile strumento per ripercorrere la bibliografia relativa al tema più aggiornata: S. Taglialagamba, 'Architetture teatrali, feste e apparati effimeri di Leonardo', in: S. Frommel \& J. Guillaume (a cura di), Leonardo e l'architettura, Modena, Panini, 2020, pp. 87-98.

49 https: / /www. leonardodigitale.com/sfoglia/codice-atlantico/0190-v/ (30 giugno 2020).
} 
da utilizzarsi per la produzione di bisantini ovvero 'lustrini' in oro, ${ }^{50}$ forse pensati per decorare gli abiti da signora, al foglio $8 \mathrm{r}$-a $[29 \text { r }]^{51}$ (c. 1493-1495) del Codice Atlantico.

Un caso eccezionale è costituito dal grande foglio di studi di tecnologia, architettura, scultura e ornato, composto da due fogli del Codice Atlantico, il $10 \mathrm{r}$-a [35 r] $]^{52}$ e il $372 \mathrm{r}-\mathrm{b}[1038 \mathrm{r}],^{53}$ e il frammento RL $12722^{54}$ di Windsor in origine parte di un'unica pagina. In basso a destra si trova il grazioso disegno di una borsa preziosa da signora: la decorazione è caratterizzata da motivi acanteiformi, disciplinati da un rigoroso impianto simmetrico. I punti di riferimento potevano essere modelli ben conosciuti come le decorazioni del monumento funebre per Giovanni e Piero de Medici in San Lorenzo realizzato dal maestro Andrea Verrocchio ma anche elementi decorativi diffusi che guardavano ai marmi di epoca augustea a decorazione vegetale. Decorazioni simili, da ricondurre alla stessa forma matrice spiraliforme, si ritrovano nella decorazione fogliacea sulla lorica militare da indossarsi come corazza per tornei o trionfi al foglio giovanile RL $12370 v^{55}$ (c. 1478-1480), e anche negli elementi vegetali sulla veste corta ricoperta da ricami tra i quali si arrampicano i tralci fogliacei di edera in un foglio ben più tardo, il RL $12281 r^{56}$ (c. 1506-1510), così come nella cosiddetta veste con i "corbezzoli" al verso dello stesso foglio che potrebbe fornire un'idea per come si presentasse quella variopinta e certa bizzarra veste che, come attestava Scipione Ammirato nel tomo II dei suoi Opuscoli editi a Firenze nel 1583, era indossata dallo stravagante Tommaso Masini detto Zoroastro che 'Si mise poi con Lionardo Vinci, il qual gli fece una veste di gallozzole, onde fu per un gran tempo nominato il Gallozzolo'. ${ }^{57}$

La decorazione a profusione rimanda al foglio del Codice Atlantico $292 \mathrm{v}$-a $[797$ r] ${ }^{58}$ (c. 1495-1497) con studi per il meccanismo di una serratura a chiavistello e la decorazione della fibbia da cintura. Nel margine superiore del foglio, rifilato, quello che sembra essere il motivo ornamentale dell'attacco a una cintura di una piccola borsa, un'insegna o un finimento, è caratterizzato dal particolare dei tre cerchi intrecciati, tenuti insieme soltanto da quello più piccolo. Come farebbe pensare la data, da collocarsi cronologicamente attorno al 1497, potrebbe riferirsi al cosiddetto nodo borromeo, simbolo inizialmente legato ai Visconti come dimostra la loro ricorrenza nelle carte dei tarocchi e poi agli Sforza come attesta la sua ricorrenza tra gli emblemi sforzeschi in un altorilievo su una vasca decorata nel cortile del Castello Sforzesco di Milano e dove ricorre anche lo stesso motivo dei girali acanteiformi. Interessante è il motivo della catena a intrecci per la quale Leonardo elabora tre maglie a volute regolari, che si complicano ulteriormente nelle tre maglie inferiori aggiungendo un'altra voluta arricciata - a testimonianza che ogni graticcio può essere

\footnotetext{
50 Tradizionalmente interpretata come una macchina per il conio di monete, è stata invece identificata in modo più corretto da Carlo Pedretti come una macchina dotata di un martello automatizzato per la produzione di listelli decorativi per abiti legando questo disegno ad altri studi: i supposti fogli gemelli, il 3 r-a [14 r] e il 393 r-d [1091 r] (c. 1493-1495) e il lievemente più tardo foglio 368 v-c [1029 v] (c. 1495), nei quali Leonardo si preoccupa di studiare rispettivamente, nei primi due, il punzone e nell'ultimo l'ossatura della struttura tecnologica della macchina. Si veda Pedretti, Codex Atlanticus of Leonardo da Vinci, cit., I, p. 34 e i fogli di riferimento.

${ }^{51}$ Cfr. https: //www.leonardodigitale.com/sfoglia/codice-atlantico/0029-r/ (30 giugno 2020).

52 Cfr. https: / /www.leonardodigitale.com/sfoglia/codice-atlantico/0035-r/ (30 giugno 2020).

${ }^{53}$ Cfr. https: / /www.leonardodigitale.com/sfoglia/codice-atlantico/1038-r/ (30 giugno 2020).

54 Cfr. https: / / www.rct.uk/collection/912722/a-sketch-of-a-nude-man (30 giugno 2020).

${ }^{55}$ Cfr. https: / /www.rct.uk/collection/912370/recto-studies-of-dragons-verso-a-design-for-a-decoratedcuirass (30 giugno 2020).

56 Cfr. https://www.rct.uk/collection/912282/recto-studies-of-emblems-decorative-dress-architectureand-a-profile-verso-studies (30 giugno 2020).

57 S. Ammirato, Opuscoli, Firenze, Nuova Stamperia D’Amadore Massi e Lorenzo Landi, 1583, tomo II, p. 242.

${ }^{58} \mathrm{Cfr}$. https: / /www.leonardodigitale.com/sfoglia/codice-atlantico/0797-r/ (30 giugno 2020).
} 
oggetto di sviluppi ulteriori ma facendo perdere all'oggetto la facilità di riproduzione. ${ }^{59}$ Lo studio di catena offre il pretesto di passare a un'altra sezione interessante: quella dei costumi per le feste e degli apparati teatrali. Amedeo Quondam nella sua introduzione al Libro del Cortegiano di Baldassarre Castiglione, afferma che 'lo spazio della Corte si identifica con lo spazio del suo teatro'. ${ }^{60} \dot{E}$ in questi disegni che l'ornato diventa pertanto un vero e proprio linguaggio.

Per l'ornato sono in particolare dei disegni tardi a interessarci. Il foglio del Codice Atlantico $133 \mathrm{r}$-a [366 r] ${ }^{61}$ (c. 1506-1508) raffigura tre else di spade: escludendo la lama, l'intento è descrivere le tre invenzioni decorative per l'elsa. In tutti i casi, il pomolo non è decorato come le restanti parti come se dovesse piuttosto rispondere alla sua funzione di bilanciamento dell'arma. La manica e la guardia di ogni disegno invece sono caratterizzate da una inventiva e una fantasia dal piglio inedito passando da una decorazione a intreccio, a quella serpentinata e ai motivi geometrici come se si procedesse con un progressivo processo di astrazione dai risultati sempre più eleganti e raffinati. Un'estrema raffinatezza interessa anche gli studi nei quali elabora idee partendo da strumenti prima esaminati da un punto di vista tecnologico e poi strutturale, come le fontane di Erone - queste, dette anche 'fontane da tavola', erano oggetti spesso realizzati in argento destinati a decorare le tavole imbandite. Il meccanismo e il funzionamento di questi dispositivi consistevano nella pressione dell'aria sull'acqua che poteva così zampillare. Nate forse in sintonia con l'allinearsi alla nuova moda francese diffusasi a seguito della calata in Italia di Carlo VIII, Leonardo inizia a interessarsi alle fontane da tavola alla fine del primo soggiorno fiorentino così come attesta la fontana a bacile schizzata al foglio $218 \mathrm{r}-\mathrm{b}$ [585 $]^{62}$ del Codice Atlantico (c. 1499-1500). Questa sarà preludio al famoso orologio d'acqua progettato per Charles d'Amboise ${ }^{63}$ e alle fontane monumentali su carta azzurra dei fogli di Windsor RL $12690^{64}$ e $12691^{65}$ (c. 1513). Si aggiungono anche per conformazione verticale a blocchi sovrapposti ma non per funzionalità, le preziose lampade da tavolo a intensità regolabile schizzate e studiate in varie fogge al foglio $283 v^{66}$ del Codice Arundel (c. 1508-1510) nelle quali l'elemento fuoco sostituisce l'acqua nello stesso modo in cui il ricorso alle pratiche tecnologiche dell'antichità permettevano di passare in maniera piuttosto sorprendente dalla tecnologia idraulica alla combustione con olio e stoppino. Proprio questi esempi mostrano che Leonardo rispecchia a pieno il suo tempo dove la pratica artigianale non subordina mai la funzionalità dell'ornato.

\section{L'ornato e i segni del potere}

È stato proposto che un gruppo di disegni tra cui i famosissimi carri falcianti (c. 1485, Torino, Biblioteca Reale, inv. 15583) fossero fogli di presentazione offerti da Leonardo a Ludovico il Moro per illustrare i punti dei paragrafi della famosa lettera scritta nel 1481, nella quale l'artista si presentava per essere scelto come ingegnere militare promettendo macchine da guerra e armi nuove e sorprendenti. Al punto 7 , in particolare, Leonardo scriveva: 'Item occurrendo di bisogno farò bombarde, mortari et passavolanti di bellissime et utile forme, fori del comune uso'. Proprio la descrizione

\footnotetext{
59 Studio per una catena a intrecci per un bracciale, c. 1490-1492, Codice Atlantico, f. 252 v-b [681 v], cfr. https: //www.leonardodigitale.com/sfoglia/codice-atlantico/0681-v/ (30 giugno 2020).

${ }^{60}$ A. Quondam \& N. Longo (a cura di), B. Castiglione, Il libro del Cortegiano, Milano, Garzanti, 1981, p. XVIII.

${ }^{61}$ Cfr. https: //www.leonardodigitale.com/sfoglia/codice-atlantico/0366-r/ (30 giugno 2020).

${ }^{62}$ Cfr. https: / /www.leonardodigitale.com/sfoglia/codice-atlantico/0585-r/ (30 giugno 2020).

63 Progetto per una fontana per Charles d'Amboise, c. 1508-10, Windsor, RL 12688 e 12716, cfr. https: //www.rct.uk/collection/912688/designs-for-a-water-clock (30 giugno 2020).

${ }^{64}$ Cfr. https: //www.rct.uk/collection/912691/designs-for-a-fountain (30 giugno 2020).

${ }^{65}$ Cfr. https: //www.rct.uk/collection/912690/designs-for-a-fountain (30 giugno 2020).

${ }^{66}$ Cfr. https: / /www.leonardodigitale.com/sfoglia/codice-arundel/0283-v/ (30 giugno 2020).
} 
'passavolanti di bellissime et utile forme, fori del comune uso' potrebbe ben applicarsi ai fogli 57 r-b [159 ii r $]^{67}$ e f. 57 v-b $\left[160\right.$ ii r] ${ }^{68}$ (c. 1485) del Codice Atlantico nei quali Leonardo schizza due tipologie diverse di lanciasassi. Sul rigore dell'immagine meccanica prevale anche il gusto dell'ornato che si esplica nel grazioso modo di atteggiare le corde in entrambi i disegni: tale motivo a ornato e a nastri svolazzanti bene si sposava a un gusto di ascendenza lombarda per accattivarsi il Signore di Milano.

In netto contrasto con queste macchine belliche 'di bellissime et utile forme' perfettamente raffigurate, al tempo stesso e in ugual misura, come una invenzione tecnologica e una creazione d'ornato, nello stesso periodo Leonardo realizza $i$ mirabolanti disegni di armi fantastiche su alcuni fogli ${ }^{69}$ del Manoscritto B (c. 1487-1490) a dimostrazione che, sbilanciando una delle due qualità di base a favore dell'altra, diventa impossibile poter parlare di oggetti che potrebbero appartenere, perlomeno concettualmente, all'idea di design. Leonardo vi raffigura armi fantastiche: le forme delle lame taglienti e affilate si fanno sempre più slanciate e sottili costringendo il materiale a negare la propria identità per raggiungere piuttosto esiti decorativi eleganti e esuberanti. Lo stesso valore hanno gli intrecci geometrici, le lame dai bordi eleganti e affilati volti a piatto, le punte uncinate e stellate: le strutture offensive di ogni arma sono solo un pretesto, è l'ornamento il vero protagonista. In questo caso specifico, infatti, viene meno l'utilità per essere potenziata al massimo grado la bellezza. Per la loro peculiare impaginazione, le armi appaiono subito non come disegni realistici ma piuttosto come immagini di repertorio, a cui verrebbe naturale associare un nome o una descrizione. Paradossalmente, l'idea grafica di squadernare a piatto le armi tende ad avvicinare queste immagini alle illustrazioni dei taccuini degli artigiani o dei quaderni da bottega anticipando perfino il gusto di un intento catalogatorio da collezione poiché disegni con simili immagini costellano gli inventari delle antiche raccolte di curiosità naturali e artificiali per il loro carattere di enciclopedia visiva. A titolo esemplificativo basti ricordare la memoria magliabechiana che documenta un'opera in lingua volgare dell'erudito Giovanni Norchiati, membro della prestigiosa Accademia degli Umidi e canonico di San Lorenzo tra il 1533 e il 1541, che elencava i nomi degli strumenti da bottega oppure il bellissimo taccuino di mano ignota con il repertorio, il nome e l'uso specifico degli strumenti usati dagli artigiani. ${ }^{70}$ In questo caso né la scelta di impaginazione né il tentativo di creare una bella immagine sono sufficienti a creare un oggetto di design ma solo un oggetto di potere. Il tentativo di persuadere e di ingraziarsi il gusto di Ludovico serviva all'artista per farsi portavoce ideale della sua politica ideando un'auto-identificazione tra i segni del potere e i simboli personali, come se questi fossero dei sigilli autocratici e autorevoli al tempo stesso. In quest'ultimo caso, l'ornato era utilizzato da Leonardo esclusivamente come segno del potere, pur declinandosi da sigillo di una autarchia imposta a una ben più pacifica e simbolica affermazione di sé in qualità di committente.

A Roma, Leonardo progetta una serie di macchine industriali per Giuliano dei Medici $^{71}$ tra cui due macchine per fabbricare corde, entrambe operate a mano ma con

\footnotetext{
${ }^{67}$ Cfr. https: / /www.leonardodigitale.com/sfoglia/codice-atlantico/0159-r/ (30 giugno 2020).

${ }^{68}$ Cfr. https: / /www.leonardodigitale.com/sfoglia/codice-atlantico/0160-r/ (30 giugno 2020).

69 Cfr. https://www.leonardodigitale.com/sfoglia/manoscritto-b-dell-institut-de-france/B001-r/ e https://www.leonardodigitale.com/sfoglia/manoscritto-b-dell-institut-de-france/B002-r/ (30 giugno 2020).

${ }^{70}$ Rispettivamente: Firenze, BNC, Ms. Magliabechi, classe IX, (c. 1540?) e Firenze, Biblioteca Medicea Laurenziana, Acquisti e Doni, 793, c. 17 r (fine XVI-inizi XVI secolo).

${ }^{71}$ A proposito del nesso tra motivi ornamentali e araldico-medicei con particolare riferimento al periodo romano, rimando al contributo fondamentale su Leonardo e Roma: D. Laurenza, Leonardo nella Roma di Leone X (c. 1513-16), XLIII Lettura Vinciana (Vinci, Biblioteca Leonardiana, 2003), Firenze, Giunti, 2004. Rimando anche al mio saggio per la nuova identificazione delle due macchine e del loro peso all'interno del progetto di fabbricazione degli specchi ustori: 'Leonardo e la scienza a Roma. Il sogno dell'unità tra
} 
sottili differenze. In due disegni, entrambi conservati nel Codice Atlantico e datati tra il 1513 e il 1515, Leonardo raffigura un'elegante cordatrice a manovella e bobina con tre ganci, a cui sono attaccati altrettanti fili posti in tensione e in torsione dal peso posto a destra al foglio $2 \mathrm{v}-\mathrm{a}[12 \mathrm{r}]^{72}$ e un torcitoio al foglio $2 \mathrm{v}-\mathrm{b}[13 \mathrm{r}]^{73}$. È quest'ultimo a interessarci: da un punto di vista strutturale, questa macchina è concepita piuttosto come una sorta di fontana con il bacino di confluenza delle acque ornato con un semplice motivo a girali. La macchina era azionata a mano ed era provvista di un congegno a vite per mantenere inalterata la tensione della cinghia di trasmissione. La vite ha la forma dell'anello dei Medici che, carico di rimandi simbolici, era usato in opere di architettura. Un esempio potrebbe essere dato dal disegno - pubblicato nel 1913 come Leonardo ma adesso attribuito a Baldassarre Peruzzi - trovato fortuitamente incollato al risguardo di copertina de Le due regole della prospettiva pratica di lacomo Barozzi da Vignola con i commentari del reverendo padre maestro Egnazio Danti, matematico dello studio di Bologna stampato a Roma nel 1611 (Lamporecchio, Nuova Fondazione Rossana e Carlo Pedretti). Pur se non avvicinabile a Leonardo, colpisce il prospetto architettonico di facciata posto sullo sfondo a punta d'argento che sembra proporre una decorazione da ricondurre all'ambiente mediceo. Gli stessi simboli, infatti, si ritroverebbero nelle insegne di Giuliano dei Medici e del fratello Giovanni, papa Leone X, come mostrano queste due incisioni contenute in Histoire des hommes illustres de la maison de Medici, avec un abbrege des comtes de Bolongne et D'Avvergne. A la roine, mere du roi, scritto da Jean Nestor e edito a Parigi nel 1564.

Il potere della bellezza diventa essa stessa il simbolo del potere. Questo vale in particolar modo per i progetti elaborati per Francesco I, caratterizzati da un gusto nuovo e raffinato. Infine, appartengono al periodo francese $i$ due disegni che raffigurano sontuosi abiti femminili per feste dai dettagli orientaleggianti studiati da Leonardo nei fogli di Windsor, RL $12576^{74}$ e RL $12577^{75}$ (c. 1517-1518). Entrambi i soggetti sono raffigurati in posizione frontale, strettamente funzionale a esibire l'elegante costume costituito da un aderente corsetto a fiocchi intrecciati decorato al centro da un clipeo circolare e dalle maniche rigonfie che sembrano trattenute a stento da nastri a varie altezze. Il corsetto ricorda la cosiddetta lorica squamata, corazza caratterizzata da lamelle di metallo disposte l'una accanto all'altra che, oltre a ricordare le squame di un pesce, aveva un grande effetto decorativo. Il corsettolorica è stretto sui fianchi da una cintura gioiello a catenelle pendenti, da cui si dipartono lembi ricamati fino all'altezza dei fianchi. Nella seconda figura invece il corsetto diventa più complicato per la fitta trama di nastri intrecciati. Così come già accadeva per i costumi, l'ornato raggiunge esiti eleganti anche in altri due esempi molto diversi tra loro: sia nello studio di elmo con cimiero a leone al foglio di Windsor RL $12329^{76}$ (c. 1517-1518), la cui profusione decorativa è strettamente funzionale alla destinazione del cimiero fantastico da indossare per una parata, sia nei leggermente più recenti disegni di artiglierie al foglio del Codice Atlantico 19 v-b [62 r] $]^{77}$ (c. 15131515) ognuno identificato da un nome e per il quale si specifica essere 'vecchio' o 'nuovo' dove colpisce il cannone decorato con il motivo del fleur de lys.

pratica e teoria', in: R. Antonelli, C. Cieri Via, A. Forcellino \& M. Forcellino (a cura di), Leonardo a Roma. Influenze ed eredità, catalogo della mostra (Roma, Villa Farnesina, 3 ottobre 2019 - 12 gennaio 2020), Roma, Bardi Edizioni, 2019, pp. 155-192, in particolare pp. 171-177.

72 https: //www.leonardodigitale.com/sfoglia/codice-atlantico/0012-r/ (30 giugno 2020).

${ }^{73}$ Cfr. https: / /www.leonardodigitale.com/sfoglia/codice-atlantico/0013-r/ (30 giugno 2020).

${ }^{74}$ Cfr. https: //www.rct.uk/collection/912576/a-standing-masquerader (30 giugno 2020).

75 Cfr. https: / /www.rct.uk/collection/912577/a-standing-masquerader (30 giugno 2020).

76 Cfr. https://www.rct.uk/collection/912329/a-design-for-a-helmet-the-head-of-a-youth-and-a-fallenhorse (30 giugno 2020).

77 Cfr. https://www.leonardodigitale.com/sfoglia/codice-atlantico/0062-r/ (30 giugno 2020). 
Per concludere, bellezza e utilità sono un binomio indissolubile e inscindibile nei disegni appena esaminati. Come indicato da Carlo Pedretti la famosa domanda 'Non può essere bellezza e utilità come appare nelle fortezze e nelli omini?' sembra essere la postilla a una lettura, individuata in un brano del De civitate Dei di sant'Agostino, libro posseduto da Leonardo nel 1504 e citato da Francesco Melzi su un foglio tardo con i progetti per Romorantin. Scrive sant'Agostino al passo 24 del libro XXIl:

Ci sono inoltre alcune cose che non hanno tal posto nel corpo, che ovviamente non hanno una funzione di utilità ma sono solo per bellezza, [...] Se perciò di tutti quei membri che sono esposti alla nostra vista, non ce n'è certo uno in cui la bellezza sia sacrificata all'utilità, mentre ce ne sono che servono solo alla bellezza, mi sembra di poter concludere che nella creazione del corpo umano la grazia ebbe precedenza sulla necessità. ${ }^{78}$

Si riabilita pertanto il binomio inscindibile bellezza e utilità tanto che la frase appuntata sul foglio del Codice Atlantico sembra perdere l'intonazione interrogativa e diventare assertiva. $\dot{E}$ in particolare a contatto con i potenti signori del tempo che Leonardo intensifica l'uso di "belle" immagini, riflesso esse stesse del volto del potere. Egli si allinea alla 'ut rethorica pictura' e alla tecnica del persuadere, definita da Aristotele rampolla della politica, declinandola in un programma visivo che avrebbe celebrato lo stato di libertà, prosperità e bellezza di quello che in realtà era un regime assolutistico.

L'ornato diventa per Leonardo un requisito essenziale alla bellezza nella misura della sua intrinseca necessità a rendere utile un elemento considerando la sua destinazione e il suo utilizzo specifico. Come se bastasse solo un segno per l'esplosione dell'emozione che, per usare una formula cara a Luca Pacioli, solo la 'gloriosa e ineffabile senistra mano' di Leonardo avrebbe potuto dare.

\section{Parole chiave}

Leonardo, industrial designer, bellezza, utilità, gioielli, mazzocchio

Sara Taglialagamba, storica dell'arte, ha dedicato i suoi studi a Leonardo e ai suoi contemporanei, in particolar modo con monografie ricche di documenti inediti su Verrocchio, su Stoldo Lorenzi e sugli orologiai Della Volpaia. Su Leonardo si occupa di settori specifici come le automazioni, la robotica, l'orologeria e l'idraulica. È stata autrice insieme a Carlo Pedretti di quattro volumi dell'Edizione Nazionale dei disegni e manoscritti di Leonardo per Giunti e Treccani. È editor in chief della serie Leonardo Studies (Brill, Leiden) e Visiting professor alla UCLA. È direttore della Nuova Fondazione Rossana e Carlo Pedretti.

Nuova Fondazione Rossana e Carlo Pedretti

Via Sambarontana 79-81

Lamporecchio (PT) (Italia)

saraart77@yahoo.it

${ }^{78}$ Si veda: Pedretti, 'Il concetto di bellezza e utilità in Sant'Agostino', cit., pp. 107-111. 


\section{Leonardo Industrial Designer}

Was Leonardo the first industrial designer? Leonardo tries to identify some matrices governing the extreme variety of things inside the whole. Such matrices are supposed to be archetypical entities which can be reproduced countless times in different ways, following geometric rules and standard proportions. We can therefore talk about a grammar of shapes that can be applied to several proportional rapports, symmetries and conceptual analogies which can generate concrete images of a stunning beauty. Leonardo was particularly keen on intertwining figures - dynamic matrices with a really high decorative potential. Leonardo makes use of twined and twisted shapes to describe very different phenomena. Matrix shapes consequently feature two different functions: they are used to investigate and better understand nature, and work as a precious tool for artistic and technical design. The decorations Leonardo creates by using them are therefore neither simple visual ornament, nor accessories to other works of art; on the contrary, they are fundamental and functional elements of a whole. Ornaments thus comply with the criteria of order, beauty and invention, which correspond to the definition of Industrial Designer formulated in the $20^{\text {th }}$ century. 